\title{
Dietary Cysteine and Other Amino Acids and Stroke Incidence in Women
}

\author{
Susanna C. Larsson, PhD; Niclas Håkansson, PhD; Alicja Wolk, DMSc
}

Background and Purpose-Cysteine could potentially lower the risk of stroke through antihypertensive and antioxidant effects. Our aim was to evaluate the hypothesis that cysteine intake is inversely associated with stroke incidence.

Methods - We used data from the Swedish Mammography Cohort, a population-based prospective cohort of 34250 women who were free of cardiovascular disease and cancer and had completed a food-frequency questionnaire about diet and other risk factors for stroke in the autumn of 1997. Stroke cases were identified by linkage of the study population with the Swedish Inpatient Register and the Swedish Cause of Death Register. Relative risks (RR) with 95\% confidence intervals, adjusted for potential confounders, were estimated by using Cox proportional hazards regression model.

Results - We ascertained 1751 incident cases of stroke during 10.4 years of follow-up. Dietary cysteine intake (mean, 635 $\mathrm{mg} / \mathrm{d}$ ) was inversely associated with stroke risk. The multivariable RR of total stroke comparing the highest with the lowest quintile of cysteine intake was 0.79 (95\% confidence interval, $0.65-0.97 ; P$ for trend $=0.04$ ). The corresponding RR was 0.82 (95\% confidence interval, $0.65-1.03 ; P$ for trend $=0.12$ ) for cerebral infarction and 0.54 (95\% confidence interval, $0.29-1.03 ; P$ for trend=0.08) for intracerebral hemorrhage. Dietary intake of other amino acids showed no independent (after adjustment for cysteine intake) association with stroke risk.

Conclusions-These findings suggest that dietary cysteine intake may be inversely associated with risk of stroke.

Clinical Trial Registration -URL: http://www.clinicaltrials.gov. Unique identifier: NCT01127698.

(Stroke. 2015;46:922-926. DOI: 10.1161/STROKEAHA.114.008022.)

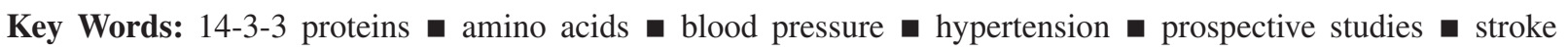

E idence from animal studies, randomized trials, and observational studies indicates that dietary protein intake is inversely associated with blood pressure ${ }^{1-3}$ and the risk of stroke. ${ }^{4,5}$ A recent meta-analysis of 7 prospective studies found that intake of total and animal protein, but not vegetable protein, was significantly inversely associated with stroke risk. ${ }^{4}$ Proteins in the diet are composed of several amino acids, each with different chemical properties. Cysteine is a nonessential sulfur-containing amino acid that may, at least in part, be responsible for the potential blood pressure and strokereducing effects of a high-protein diet. It is more abundant in animal and cereal proteins than in legume proteins. ${ }^{6}$ Foods rich in cysteine include poultry, egg, beef, and whole grains. ${ }^{6}$ Cysteine may exert its antihypertensive effects directly or via its storage form, glutathione, by reducing oxidative stress and advanced glycation end products, improving insulin sensitivity and glucose metabolism, and modulating nitric oxide levels. ${ }^{7}$ To the best of our knowledge, no study has examined the association between cysteine intake and risk of stroke.

In a previous study, based on data from the Swedish Mammography Cohort (SMC), we observed inverse associations of total and animal protein intakes with stroke incidence. ${ }^{8}$
In this study, we evaluated the a priori hypothesis that cysteine intake is inversely associated with risk of stroke, and that cysteine may have contributed to our previous observed finding for dietary protein. We also provide results for other amino acids, most of which are highly correlated with protein intake, in relation to stroke risk.

\section{Methods}

\begin{abstract}
Study Population
This study was based on data from the SMC, which is a populationbased prospective cohort study of Swedish women. ${ }^{9}$ In the autumn of 1997, 56030 participants of the SMC obtained a comprehensive questionnaire that included $\approx 350$ items on diet, other lifestyle factors, etc; 39227 women (70\%) returned a completed questionnaire. We excluded women with an erroneous or a missing personal identification number $(n=243)$, women who did before start of follow-up (January 1, 1998; $\mathrm{n}=26$ ), women with a diagnosis of cardiovascular disease $(n=2492)$ or cancer $(n=1811)$ before start of follow-up, and women with an implausibly high or low energy intake (ie, 3 SDs from the $\log _{\mathrm{e}}$-transformed mean energy intake; $n=405$ ). After exclusions, 34250 women aged $49-83$ years remained for analysis. The study was approved by the Regional Ethical Review Board at the Karolinska Institutet in Stockholm.
\end{abstract}

Received November 4, 2014; final revision received January 12, 2015; accepted January 15, 2015.

From the Unit of Nutritional Epidemiology, Institute of Environmental Medicine, Karolinska Institutet, Stockholm, Sweden.

Correspondence to Susanna C. Larsson, PhD, Unit of Nutritional Epidemiology, Institute of Environmental Medicine, Karolinska Institutet, Box 210, SE-17177 Stockholm, Sweden. E-mail susanna.larsson@ki.se

(C) 2015 American Heart Association, Inc. 


\section{Baseline Data Collection}

The questionnaire inquired about education level, body weight and height, smoking status and history, physical activity, aspirin use, history of diabetes mellitus and hypertension, family history of myocardial infarction before 60 years of age, alcohol consumption, and diet. Participants were classified as having diabetes mellitus if they had a diabetes mellitus diagnosis recorded in the Swedish National Inpatient Register or the Swedish National Diabetes Register, or reported in the questionnaire that they had diabetes mellitus. History of hypertension was based on self-report only. We computed body mass index (BMI) as the weight in kilograms divided by the square of height in meters. Pack-years of smoking history were calculated as the number of packs of cigarettes smoked daily multiplied by the number of years of smoking. The physical activity questions included level of activity at work, home/housework, walking/bicycling, and exercise. There were also questions about inactivity, including watching TV/reading, sleeping, and sitting/lying down. The reported time spent on every activity was multiplied by the activity's typical energy expenditure requirement expressed in metabolic equivalents (MET), and added up to create a MET-hours per day (24 hours) score. ${ }^{10}$

\section{Dietary Assessment}

Diet was assessed with a 96-item food-frequency questionnaire on which participants reported how often, on average, they had consumed various foods/food items during the past year. There were 8 predefined frequency categories ranging from never to $\geq 3 \times$ times per day. Intake of amino acids and other nutrients was calculated by multiplying the frequency of intake of each food by the nutrient content of age-specific ( $<53,53-65$, and $\geq 66$ years) portion sizes, using composition values from the Swedish Food Administration Database. All amino acids and nutrients (except alcohol) were adjusted for total energy intake by using the residual method. ${ }^{11}$ The food-frequency questionnaire has been validated, and the Spearman correlation coefficients between estimates from the questionnaire and the average of fourteen 24 -hour recall interviews was 0.44 for dietary protein. ${ }^{12}$

\section{Case Ascertainment and Follow-Up}

By linkage of the study population to the Swedish Inpatient Register and the Swedish Cause of Death Register, we obtained information on stroke events and stroke deaths that occurred in the study cohort. Diagnoses in the Swedish Inpatient Register are coded according to the Swedish International Classification of Disease system (adapted from the World Health Organization International Classification of Disease classification system). We classified the strokes as cerebral infarctions (International Classification of Disease-Tenth Revision code I63), intracerebral hemorrhages (I61), subarachnoid hemorrhages (I60), and unspecified strokes (I64). We received information on dates of death from the Swedish Death Registry.

\section{Statistical Methods}

Follow-up time for each participant was computed from January 1,1998 to the date of first stroke event, death, or end of follow-up (December 31, 2008), whichever occurred first. Cox proportional hazard regression models with age as the time scale was used to estimate relative risks (RRs) and 95\% confidence intervals (CIs) of stroke by quintiles of amino acid intake. Entry time was defined as a woman's age in months at start of follow-up, and exit time was defined as a woman's age in months at censoring. The proportional hazards assumption was tested and was found to be met. In a first multivariable model, we adjusted for a combination of smoking status and pack-years of smoking (never [reference]; past $<20,20-39$, or $\geq 40$ pack-years; or current $<20,20-39$, or $\geq 40$ pack-years), education (less than high school, high school, or university), BMI $(<20,20-24.9$ [reference], 25-29.9, or $\geq 30 \mathrm{~kg} / \mathrm{m}^{2}$ ), total physical activity (quartiles), history of hypertension (yes or no), history of diabetes mellitus (yes or no), aspirin use (yes or no), family history of myocardial infarction before 60 years of age (yes or no), total energy intake (continuous in $\mathrm{kcal} / \mathrm{d}$ ), and quintiles of alcohol and dietary cholesterol intake. In a second multivariable model, we further controlled for quintiles of total protein intake. Adjustment for dietary fiber and nutrients, including dietary fat, saturated fat, carbohydrates, vitamin $\mathrm{C}$, dietary folate, magnesium, potassium, and calcium did not change the results materially. Therefore, those nutrients were not included in the final model. Likewise, we did not include history of hypercholesterolemia

Table 1. Age-Standardized Baseline Characteristics* of 34250 Women in the Swedish Mammography Cohort by Quintiles of Energy-Adjusted Cysteine Intake

\begin{tabular}{lccccc}
\hline & \multicolumn{5}{c}{ Cysteine Intake, mg/d } \\
\cline { 2 - 6 } & $<559(524) \dagger$ & $559-603(583)$ & $604-645(624)$ & $646-702(671)$ & $\geq 703(755)$ \\
\hline No. of women & 6849 & 6852 & 6863 & 6831 & 6855 \\
Age, y & 60.7 & 60.5 & 61.0 & 61.5 & 62.5 \\
Education, university, \% & 18.9 & 18.6 & 19.9 & 20.1 & 20.8 \\
Current smoker, \% & 27.4 & 27.5 & 22.4 & 21.2 & 21.4 \\
Body mass index, kg/m ${ }^{2}$ & 24.4 & 24.8 & 24.9 & 25.2 & 25.7 \\
Total physical activity, MET-h/d & 42.3 & 42.7 & 42.7 & 42.7 & 42.6 \\
History of hypertension, \% & 16.6 & 18.0 & 19.2 & 20.8 & 23.4 \\
Diabetes mellitus, \% & 1.57 & 2.09 & 2.41 & 3.93 & 7.59 \\
Aspirin, any use, \% & 49.5 & 48.8 & 49.6 & 50.0 & 48.8 \\
Family history of myocardial & 15.2 & 16.0 & 16.0 & 17.0 & 18.6 \\
infarction, \% & & & & 5.86 \\
Alcohol consumption, g/d & 8.49 & 6.84 & 1720 & 1710 & 5.61 \\
Total energy intake, kcal/d & 1770 & 1750 & 69.9 & 73.5 & 1770 \\
Total protein intake, g/d & 59.8 & 66.5 & 233 & 237 & 83.4 \\
Dietary cholesterol intake, mg/d & 216 & 229 & 253 \\
\hline
\end{tabular}

MET indicates metabolic equivalent of energy expenditure (kcal/[kg h]).

*Values are means if not otherwise indicated.

†Median values in parenthesis. 
and hormone replacement therapy use in the final model because adjustment for those variables did not alter the results.

We performed tests for trend by assigning the median value of cysteine intake to each quintile and modeled this variable as a continuous variable. Tests for interaction between cysteine intake and history of hypertension (yes or no) or age (median split; $<60$ versus $\geq 60$ years of age) in relation to stroke risk were conducted by using the likelihood ratio test. All analyses were performed using SAS, version 9.3 (SAS Institute Inc, Cary, NC). All statistical tests were 2-tailed; $P<0.05$ was considered statistically significant.

\section{Results}

Baseline characteristics of study participants according to quintiles of dietary cysteine intake are shown in Table 1 . Mean $( \pm$ SD) intake of cysteine was $635( \pm 101) \mathrm{mg} / \mathrm{d}$. Compared with women in the lowest quintile of cysteine intake, those in the highest quintile tended to be older, had a higher BMI, and were less likely to be current smoker but more likely to have a history of hypertension, a history of diabetes mellitus, and a family history of myocardial infarction. In addition, they consumed less alcohol and had higher intakes of protein and cholesterol. The Spearman correlation coefficient between intake of individual amino acids $(n=18)$ and total protein was $>0.90$ for all amino acids except cysteine $(r=0.71)$, glycine $(r=0.77)$, proline $(r=0.87)$, and arginine $(r=0.88)$. Cysteine intake was more correlated with intake of animal protein $(r=0.50)$ than vegetable protein $(r=0.32)$.

We ascertained 1751 incident cases of first stroke during a mean follow-up of 10.4 years (355396 person-years). The stroke cases included 1311 cerebral infarctions, 169 intracerebral hemorrhages, 95 subarachnoid hemorrhages, and 176 unspecified strokes. The association between cysteine intake and risk of total stroke and stroke types are presented in Table 2. Cysteine intake was statistically significantly inversely associated with risk of total stroke after adjustment for other stroke risk factors and after additional adjustment for total protein intake. A high cysteine intake was associated with a reduced risk of cerebral infarction, intracerebral hemorrhage, and total hemorrhagic stroke, but none of the associations were statistically significant after further adjustment for protein intake (model 2).

The Spearman correlation coefficients between intake of cysteine and other amino acids ranged from 0.51 for proline to 0.84 for arginine (Table 3 ). Intake of most amino acids was statistically significantly inversely associated with total stroke risk after adjustment for other risk factors (multivariable model 1), but none of these associations persisted after additional adjustment for dietary cysteine (Table 3). The inverse association between cysteine intake and stroke remained (multivariable RR for highest versus lowest quintile ranged

Table 2. Relative Risks (95\% Confidence Intervals) of Total Stroke and Stroke Types by Quintiles of Energy-Adjusted Cysteine Intake in the Swedish Mammography Cohort $(n=34250), 1998$ to 2008

\begin{tabular}{|c|c|c|c|c|c|c|}
\hline & \multicolumn{5}{|c|}{ Quintile of Cysteine Intake, mg/d } & \multirow[b]{2}{*}{$P$-Trend } \\
\hline & $<559$ & $559-603$ & $604-645$ & $646-702$ & $\geq 703$ & \\
\hline \multicolumn{7}{|l|}{ Total stroke } \\
\hline Person-years & 70698 & 71328 & 71729 & 70825 & 70816 & \\
\hline No. of cases & 363 & 334 & 301 & 375 & 378 & \\
\hline Age-adjusted model & 1.00 & $0.95(0.82-1.10)$ & $0.80(0.69-0.94)$ & $0.97(0.84-1.12)$ & $0.88(0.76-1.02)$ & 0.16 \\
\hline Multivariable model $1^{*}$ & 1.00 & $0.94(0.80-1.09)$ & $0.77(0.66-0.90)$ & $0.91(0.79-1.06)$ & $0.77(0.66-0.89)$ & 0.0009 \\
\hline Multivariable model $2 \dagger$ & 1.00 & $0.96(0.82-1.13)$ & $0.81(0.68-0.96)$ & $0.95(0.80-1.14)$ & $0.79(0.65-0.97)$ & 0.04 \\
\hline \multicolumn{7}{|l|}{ Cerebral infarction } \\
\hline No. of cases & 262 & 254 & 223 & 288 & 284 & \\
\hline Age-adjusted model & 1.00 & $1.00(0.85-1.19)$ & $0.82(0.69-0.98)$ & $1.02(0.87-1.21)$ & $0.91(0.77-1.07)$ & 0.36 \\
\hline Multivariable model $1^{*}$ & 1.00 & $0.98(0.82-1.16)$ & $0.78(0.65-0.93)$ & $0.94(0.79-1.12)$ & $0.76(0.64-0.90)$ & 0.003 \\
\hline Multivariable model $2 \dagger$ & 1.00 & $1.01(0.84-1.21)$ & $0.82(0.67-1.00)$ & $0.99(0.80-1.20)$ & $0.82(0.65-1.03)$ & 0.12 \\
\hline \multicolumn{7}{|l|}{ Intracerebral hemorrhage } \\
\hline No. of cases & 38 & 32 & 32 & 34 & 33 & \\
\hline Age-adjusted model & 1.00 & $0.86(0.54-1.38)$ & $0.81(0.51-1.30)$ & $0.84(0.53-1.34)$ & $0.75(0.47-1.20)$ & 0.26 \\
\hline Multivariable model $1^{*}$ & 1.00 & $0.88(0.55-1.42)$ & $0.83(0.52-1.34)$ & $0.88(0.55-1.41)$ & $0.76(0.47-1.23)$ & 0.30 \\
\hline Multivariable model $2 \dagger$ & 1.00 & $0.81(0.49-1.34)$ & $0.73(0.43-1.25)$ & $0.75(0.43-1.30)$ & $0.54(0.29-1.03)$ & 0.08 \\
\hline \multicolumn{7}{|l|}{ Total hemorrhagic strokeł } \\
\hline No. of cases & 61 & 52 & 47 & 53 & 51 & \\
\hline Age-adjusted model & 1.00 & $0.86(0.59-1.24)$ & $0.75(0.51-1.09)$ & $0.83(0.57-1.20)$ & $0.75(0.52-1.09)$ & 0.16 \\
\hline Multivariable model $1^{\star}$ & 1.00 & $0.88(0.60-1.27)$ & $0.76(0.52-1.12)$ & $0.87(0.60-1.27)$ & $0.77(0.52-1.13)$ & 0.22 \\
\hline Multivariable model $2 \dagger$ & 1.00 & $0.82(0.56-1.22)$ & $0.69(0.45-1.06)$ & $0.76(0.49-1.18)$ & $0.60(0.36-1.01)$ & 0.07 \\
\hline
\end{tabular}


Table 3. Multivariable Relative Risks ( $95 \%$ Confidence Intervals) of Total Stroke by Energy-Adjusted Amino Acid Intake in the Swedish Mammography Cohort ( $\mathrm{n}=34250), 1998$ to 2008

\begin{tabular}{|c|c|c|c|c|c|}
\hline \multirow[b]{2}{*}{ Amino Acid } & \multirow[b]{2}{*}{ Mean Intake, $\mathrm{mg} / \mathrm{d}$} & \multirow[b]{2}{*}{ SD } & \multirow{2}{*}{$\begin{array}{l}\text { Correlation With Cysteine } \\
\text { Intake }\end{array}$} & \multicolumn{2}{|c|}{ Highest vs Lowest Quintile } \\
\hline & & & & Model $1^{*}$ & Model $2 \dagger$ \\
\hline Glutamic acid & 12779 & 2121 & 0.70 & $0.83(0.72-0.97)$ & $0.96(0.79-1.16)$ \\
\hline Aspartic acid & 5661 & 921 & 0.74 & $0.79(0.68-0.92)$ & $0.89(0.73-1.09)$ \\
\hline Leucine & 5486 & 1067 & 0.65 & $0.84(0.72-0.98)$ & $0.96(0.80-1.16)$ \\
\hline Proline & 5387 & 1130 & 0.51 & $0.89(0.76-1.03)$ & $0.99(0.84-1.17)$ \\
\hline Lysine & 4695 & 966 & 0.63 & $0.89(0.76-1.04)$ & $1.03(0.86-1.25)$ \\
\hline Valine & 4263 & 805 & 0.62 & $0.84(0.72-0.98)$ & $0.96(0.80-1.15)$ \\
\hline Serine & 3346 & 596 & 0.70 & $0.84(0.72-0.98)$ & $0.98(0.81-1.20)$ \\
\hline Isoleucine & 3282 & 632 & 0.67 & $0.86(0.74-1.00)$ & $1.00(0.82-1.21)$ \\
\hline Arginine & 3189 & 559 & 0.84 & $0.86(0.74-1.00)$ & $1.13(0.88-1.45)$ \\
\hline Phenylalanine & 3161 & 561 & 0.70 & $0.85(0.73-0.99)$ & $1.01(0.82-1.23)$ \\
\hline Alanine & 2992 & 560 & 0.82 & $0.83(0.71-0.97)$ & $1.04(0.82-1.33)$ \\
\hline Threonine & 2575 & 482 & 0.74 & $0.88(0.75-1.02)$ & $1.07(0.87-1.31)$ \\
\hline Tyrosine & 2528 & 576 & 0.52 & $0.88(0.75-1.03)$ & $0.99(0.83-1.18)$ \\
\hline Glycine & 2499 & 439 & 0.82 & $0.83(0.71-0.96)$ & $0.99(0.78-1.25)$ \\
\hline Histidine & 1794 & 331 & 0.67 & $0.89(0.76-1.04)$ & $1.08(0.89-1.32)$ \\
\hline Methionine & 1597 & 336 & 0.61 & $0.85(0.73-0.99)$ & $0.97(0.80-1.17)$ \\
\hline Tryptophan & 808 & 137 & 0.66 & $0.83(0.71-0.96)$ & $0.95(0.78-1.15)$ \\
\hline
\end{tabular}

${ }^{*}$ Model 1 was adjusted for age, smoking status and pack-years of smoking, education, body mass index, total physical activity, history of hypertension, history of diabetes mellitus, aspirin use, family history of myocardial infarction, and intakes of total energy, alcohol, and dietary cholesterol.

†Model 2 was adjusted for the same variables as model 1 and further for quintiles of cysteine intake.

from 0.70 to 0.83 ) in multivariable models adjusted for any of the other 17 amino acids.

We next examined whether dietary cysteine may account for our previously observed inverse relation between total protein intake and risk of stroke. ${ }^{8}$ The multivariable (model 1) RR of total stroke for the highest versus lowest quintile of protein intake was 0.82 (95\% CI, 0.70-0.95). This association did not remain after adding cysteine intake (in quintiles) to the model (corresponding RR=0.95; 95\% CI, 0.77-1.16).

Finally, we examined whether the association between cysteine intake and risk of stroke was modified by history of hypertension. The multivariable (model 2) RRs of total stroke for the highest compared with the lowest quintile of cysteine intake were 0.75 (95\% CI, 0.59-0.96) in women without hypertension at baseline and 0.88 (95\% CI, 0.62-1.25) in those with hypertension ( $P$ for interaction $=0.07$ ). We found no interaction between cysteine intake and age in relation to stroke risk ( $P$ for interaction $=0.70)$.

\section{Discussion}

Findings from this prospective study in women suggest that a high cysteine intake may lower the risk of stroke. Women in the highest quintile of cysteine intake had a $21 \%$ lower risk of stroke compared with those in the lowest quintile. Our results further suggest that dietary cysteine may account, at least in part, for our previous observed inverse associations of total protein and animal protein intake with risk of stroke. ${ }^{8}$ None of the other 17 amino acids was independently associated with stroke risk.
Rich food sources of cysteine are poultry, egg, beef, and whole grains. ${ }^{6}$ As consumption of red meat and processed meat is positively associated with risk of stroke, ${ }^{13}$ dietary cysteine should preferably be obtained from nonmeat sources.

A reduction in blood pressure and thus, in the risk of stroke by an increased cysteine intake is biologically plausible. It has been proposed that cysteine and its storage form, glutathione, could exert antihypertensive effects by modulating the levels of nitric oxide (NO) and other vasoactive molecules, as well as by reducing oxidative stress, insulin resistance, and advanced glycation products. ${ }^{7}$ Although cysteine is one of the least abundant amino acids, it often occurs in the functional sites of proteins. ${ }^{14}$ For example, cysteine is a component of endothelial nitric oxide synthase, ${ }^{15}$ which catalyzes the conversion of $\mathrm{L}$-arginine to NO. NO is involved in the regulation of vascular tone. ${ }^{16}$ Several studies have shown that dietary supplementation with the cysteine derivate $N$-acetylcysteine (NAC) can prevent or attenuate increased blood pressure in animal models of hypertension. ${ }^{7,17}$ In addition, using NAC as an adjunct to other hypertensive therapies leads to a decrease in blood pressure in humans. ${ }^{7,18}$ The availability of cysteine is a major determinant of the biosynthesis of glutathione, which is an important antioxidant. ${ }^{19}$ Hence, cysteine may, in part, lower stroke risk by a reduction in oxidative stress.

In this study, the inverse association between cysteine intake and stroke seemed to be stronger in women without hypertension at baseline. The weaker association in those with hypertension might be due to the antihypertensive effects of cysteine that are diminished by the use of antihypertensive drugs. 
To our best knowledge, there are no previous epidemiological studies on dietary amino acids in relation to stroke risk, but the Rotterdam Study ${ }^{20}$ and the International Collaborative Study of Macronutrients, Micronutrients, and Blood Pressure (INTERMAP) $)^{21,22}$ examined the association between intake of certain amino acids (as a percentage of total protein intake) and blood pressure. The Rotterdam Study suggested no major role for cysteine, glutamic acid, arginine, lysine or tyrosine intake in determining blood pressure in a cross-sectional analysis. ${ }^{20}$ There was also no association between intake of the 5 amino acids and risk of developing hypertension during 6 years of follow-up. ${ }^{20}$ Mean cysteine intake was much higher in the Rotterdam Study ( $\approx 600 \mathrm{mg}$ cysteine per $1000 \mathrm{kcal}$ ) than in our study ( $\approx 360 \mathrm{mg}$ cysteine per $1000 \mathrm{kcal})$. Increased cysteine intake may only have an effect in those with low intakes. In cross-sectional analyses of the INTERMAP, glutamic acid intake was inversely ${ }^{21}$ and glycine intake positively ${ }^{22}$ associated with blood pressure, whereas no association was observed for phenylalanine, proline, cysteine, or serine intake. ${ }^{21}$ The positive association of dietary glycine with blood pressure was stronger in Western participants, where meat was the main source of glycine, than in East Asian participants, where the main sources were grains/flour, rice/noodles, and fish/shellfish. ${ }^{22}$

Major strengths of this study are the prospective and population-based design and the large number of incident stroke cases. Limitations include the use of a self-administered questionnaire to assess diet and other stroke risk factors. Given the prospective design, any measurement error in the assessment of amino acid intake would most probably be unrelated to the outcome (stroke). Nondifferential misclassification of cysteine intake may have resulted in attenuated risk estimates. Another limitation is that information on stroke diagnoses was obtained from the Swedish Inpatient Register and the Cause of Death Register, which are not $100 \%$ complete. ${ }^{23}$ Thus, there may be some misclassification of stroke cases. Finally, because of the observational design of the study, we cannot rule out that the observed findings are explained by confounding because of measurement error in the variables that we adjusted for or to unmeasured confounders. For example, we did not have information on family history of stroke. Furthermore, we cannot exclude the possibility that cysteine intake represents a certain dietary pattern.

In conclusion, we observed an inverse association between dietary cysteine intake and risk of stroke. Our findings further suggest that cysteine, at least partly, may account for the reported inverse relation between animal protein intake and stroke risk. ${ }^{4}$ These results warrant confirmation by other prospective studies and randomized trials.

\section{Sources of Funding}

This study was supported by a research grant from the Swedish Research Council.

\section{Disclosures}

None.

\section{References}

1. Obarzanek E, Velletri PA, Cutler JA. Dietary protein and blood pressure. JAMA. 1996;275:1598-1603.
2. Altorf-van der Kuil W, Engberink MF, Brink EJ, van Baak MA, Bakker SJ, Navis G, et al. Dietary protein and blood pressure: a systematic review. PLoS One. 2010;5:e12102. doi: 10.1371/journal.pone.0012102.

3. Appel LJ, Sacks FM, Carey VJ, Obarzanek E, Swain JF, Miller ER III, et al; OmniHeart Collaborative Research Group. Effects of protein, monounsaturated fat, and carbohydrate intake on blood pressure and serum lipids: results of the OmniHeart randomized trial. JAMA. 2005;294:2455-2464. doi: 10.1001/jama.294.19.2455.

4. Zhang Z, Xu G, Yang F, Zhu W, Liu X. Quantitative analysis of dietary protein intake and stroke risk. Neurology. 2014;83:19-25. doi: 10.1212/ WNL.0000000000000551.

5. Chiba T, Itoh T, Tabuchi M, Satou T, Ezaki O. Dietary protein, but not carbohydrate, is a primary determinant of the onset of stroke in strokeprone spontaneously hypertensive rats. Stroke. 2009;40:2828-2835. doi: 10.1161/STROKEAHA.109.555383.

6. Stipanuk MH. Homocystein, cysteine, and taurine. In: Shils M, Olson J, Shike M, Ross AC, eds. Modern Nutrition in Health and Disease. 9th ed. Baltimore, MD: Williams \& Wilkins; 1999:543-558.

7. Vasdev S, Singal P, Gill V. The antihypertensive effect of cysteine. Int J Angiol. 2009;18:7-21.

8. Larsson SC, Virtamo J, Wolk A. Dietary protein intake and risk of stroke in women. Atherosclerosis. 2012;224:247-251. doi: 10.1016/j. atherosclerosis.2012.07.009.

9. Wolk A, Bergström R, Hunter D, Willett W, Ljung H, Holmberg L, et al. A prospective study of association of monounsaturated fat and other types of fat with risk of breast cancer. Arch Intern Med. 1998;158:41-45.

10. Norman A, Bellocco R, Bergström A, Wolk A. Validity and reproducibility of self-reported total physical activity-differences by relative weight. Int J Obes Relat Metab Disord. 2001;25:682-688. doi: 10.1038/ sj.ijo.0801597.

11. Willett W, Stampfer MJ. Total energy intake: implications for epidemiologic analyses. Am J Epidemiol. 1986;124:17-27.

12. Messerer M, Johansson SE, Wolk A. The validity of questionnaire-based micronutrient intake estimates is increased by including dietary supplement use in Swedish men. J Nutr. 2004;134:1800-1805.

13. Kaluza J, Wolk A, Larsson SC. Red meat consumption and risk of stroke: a meta-analysis of prospective studies. Stroke. 2012;43:2556-2560. doi: 10.1161/STROKEAHA.112.663286.

14. Marino SM, Gladyshev VN. Cysteine function governs its conservation and degeneration and restricts its utilization on protein surfaces. $J \mathrm{Mol}$ Biol. 2010;404:902-916. doi: 10.1016/j.jmb.2010.09.027.

15. Chen PF, Tsai AL, Wu KK. Cysteine 184 of endothelial nitric oxide synthase is involved in heme coordination and catalytic activity. $J$ Biol Chem. 1994;269:25062-25066.

16. Forstermann U, Sessa WC. Nitric oxide synthases: regulation and function. Eur Heart J. 2012;33:829-837, 837a-837d. doi: 10.1093/eurheartj/ ehr304.

17. Pechánová O, Zicha J, Kojsová S, Dobesová Z, Jendeková L, Kunes J. Effect of chronic N-acetylcysteine treatment on the development of spontaneous hypertension. Clin Sci (Lond). 2006;110:235-242. doi: 10.1042/CS20050227.

18. Martina V, Masha A, Gigliardi VR, Brocato L, Manzato E, Berchio $\mathrm{A}$, et al. Long-term $\mathrm{N}$-acetylcysteine and L-arginine administration reduces endothelial activation and systolic blood pressure in hypertensive patients with type 2 diabetes. Diabetes Care. 2008;31:940-944. doi: $10.2337 / \mathrm{dc} 07-2251$.

19. Lu SC. Regulation of hepatic glutathione synthesis: current concepts and controversies. FASEB J. 1999;13:1169-1183.

20. Altorf-van der Kuil W, Engberink MF, De Neve M, van Rooij FJ, Hofman A, van't Veer P, et al. Dietary amino acids and the risk of hypertension in a Dutch older population: the Rotterdam Study. Am J Clin Nutr. 2013;97:403-410. doi: 10.3945/ajcn.112.038737.

21. Stamler J, Brown IJ, Daviglus ML, Chan Q, Kesteloot H, Ueshima $\mathrm{H}$, et al; INTERMAP Research Group. Glutamic acid, the main dietary amino acid, and blood pressure: the INTERMAP Study (International Collaborative Study of Macronutrients, Micronutrients and Blood Pressure). Circulation. 2009;120:221-228. doi: 10.1161/ CIRCULATIONAHA.108.839241.

22. Stamler J, Brown IJ, Daviglus ML, Chan Q, Miura K, Okuda N, et al. Dietary glycine and blood pressure: the International Study on Macro/ Micronutrients and Blood Pressure. Am J Clin Nutr. 2013;98:136-145. doi: 10.3945/ajen.112.043000.

23. Appelros P, Terént A. Validation of the Swedish inpatient and causeof-death registers in the context of stroke. Acta Neurol Scand. 2011;123:289-293. doi: 10.1111/j.1600-0404.2010.01402.x. 